\title{
Suppression of carbonic anhydrase IX leads to aberrant focal adhesion and decreased invasion of tumor cells
}

\author{
PETER RADVAK $^{1}$, MARKO REPIC $^{1,4}$, ELISKA SVASTOVA ${ }^{1}$, MARTINA TAKACOVA ${ }^{1,2}$, \\ LUCIA CSADEROVA $^{1,2}$, HYNEK STRNAD ${ }^{3}$, JAROMIR PASTOREK ${ }^{1}$, \\ SILVIA PASTOREKOVA ${ }^{1}$ and JURAJ KOPACEK ${ }^{1}$ \\ ${ }^{1}$ Institute of Virology, Department of Molecular Medicine, Slovak Academy of Sciences, 84505 Bratislava; \\ ${ }^{2}$ Centre for Molecular Medicine, Slovak Academy of Sciences, 83101 Bratislava, Slovak Republic; \\ ${ }^{3}$ Institute of Molecular Genetics, Academy of Sciences of the Czech Republic, 14220 Prague 4, Czech Republic
}

Received November 6, 2012; Accepted December 5, 2012

DOI: $10.3892 / o r .2013 .2226$

\begin{abstract}
Carbonic anhydrase IX (CA IX) is a well-recognized hypoxia marker with promising diagnostic and therapeutic value. CA IX regulates the $\mathrm{pH}$ in hypoxic tumor cells and, thereby, contributes to microenvironmental acidosis and cell migration. To gain a better insight into the molecular processes driven by CA IX, we performed gene expression profiling of HT-1080 fibrosarcoma cells subjected to CA IX depletion by shRNA silencing. We identified the focal adhesion pathway as being significantly inhibited in the absence of CA IX and confirmed this finding by functional assays. Thus, we obtained the first direct evidence for the role of CA IX in focal adhesion.
\end{abstract}

\section{Introduction}

Growing tumors often develop areas of hypoxia due to an insufficient supply of oxygen as a consequence of irregular and functionally defective vasculature. Hypoxia then creates selective pressure resulting in the expansion of adaptable cells with a more aggressive phenotype and increased metastatic potential. The primary molecular response to low oxygen is stabilization and activation of an $\alpha$ subunit of hypoxia-inducible factor (HIF), a key transactivator of genes involved in the

Correspondence to: Dr Juraj Kopacek, Institute of Virology, Department of Molecular Medicine, Slovak Academy of Sciences, Dubravska cesta 9, 84505 Bratislava, Slovak Republic

E-mail: virukopa@savba.sk

Present address: ${ }^{4} \mathrm{IMBA}$ - Institute of Molecular Biotechnology GmbH, Dr. Bohr-Gasse 3, 1030 Vienna, Austria

Abbreviations: CA IX, carbonic anhydrase IX protein; $C A 9$, carbonic anhydrase 9 gene or mRNA; dox, doxycycline; FA, focal adhesion; GFP, green fluorescent protein; HIF, hypoxia-inducible factor; MMP, matrix metalloproteinase; MOI, multiplicity of infection; Scr, scrambled; shRNA, short hairpin RNA

Key words: carbonic anhydrase IX, hypoxia, shRNA silencing, microarray, focal adhesion adaptation to hypoxia, including genes participating in growth arrest, apoptosis, anaerobic glycolysis, angiogenesis, acidosis, cell adhesion and cell proliferation (1). Carbonic anhydrase IX (CA IX) is one of the most prominent hypoxia-inducible HIF-target molecules (2-4). It belongs to the family of carbonic anhydrases, which are zinc-binding enzymes that catalyze the reversible conversion of carbon dioxide to bicarbonate and protons in a reaction that involves facilitated hydration of $\mathrm{CO}_{2}$ to $\mathrm{H}_{2} \mathrm{CO}_{3}$ followed by spontaneous dissociation of $\mathrm{H}_{2} \mathrm{CO}_{3}$ to bicarbonate and protons (2). Via this catalytic activity, carbonic anhydrases significantly contribute to the modulation of ion transport and maintenance of an acid-base balance in cells and tissues of virtually all living organisms. The CA IX isoform is a highly active transmembrane glycoprotein, which differs from other carbonic anhydrases by a predominant association with various types of tumors, including carcinomas of the cervix uteri, kidney, lung, colon, breast, brain and ovary (5).

A high catalytic performance predisposes CA IX to function in ion transport and $\mathrm{pH}$ control, and tumor cells efficiently utilize this ability of CA IX to resist a hostile microenvironment. Indeed, CA IX helps to maintain intracellular $\mathrm{pH}$ of cancer cells within the physiological range, while simultaneously contributing to the acidification of the extracellular space $(6,7)$. This has important implications for cancer progression since a neutral intracellular $\mathrm{pH}$ is vital for cell proliferation and survival, whereas microenvironmental acidosis contributes to an aggressive tumor phenotype by promoting invasion and metastasis (8). In addition, CA IX decreases E-cadherin-mediated cell-cell adhesion through the competitive binding to $\beta$-catenin (9), and increases cell migration via physical interaction and functional cooperation with bicarbonate transporters in lamellipodia of moving cells (10). This further supports the pro-metastatic propensity of tumor cells.

To better understand the role of CA IX in tumor cells and disclose the underlying molecular mechanisms, we developed a CA9 mRNA-targeting shRNA inducible system and studied changes in the gene expression profile connected with CA IX protein deficiency in the HT-1080 tumor cell line. Using this approach, we identified a number of potential CA IX downstream targets belonging to several cancer-related pathways. 
Among them, the focal adhesion pathway was one of the most prominent, and thus, we focused on CA IX involvement in focal adhesion-associated phenomena.

\section{Materials and methods}

Cell culture. HT-1080, HEK 293T and HeLa cells were cultivated in DMEM with 10\% FCS (Lonza BioWhittaker) at $37^{\circ} \mathrm{C}$ in humidified air with $5 \% \mathrm{CO}_{2}$. Hypoxic experiments were performed in an anaerobic workstation (Ruskinn Technologies) in a $2 \% \mathrm{O}_{2}, 2 \% \mathrm{H}_{2}, 5 \% \mathrm{CO}_{2}, 91 \% \mathrm{~N}_{2}$ atmosphere at $37^{\circ} \mathrm{C}$. The conditional shRNA system in HT-1080 cells was activated with $0.5 \mu \mathrm{g} / \mathrm{ml}$ doxycycline (Clontech, Mountain View, CA, USA) in cultivation medium.

Antibodies and plasmids. Anti-CA IX mouse monoclonal antibody M75 was characterized elsewhere (3). Additional antibodies were as follows: goat polyclonal anti-actin antibody (Santa Cruz Biotechnology, Santa Cruz, CA, USA), mouse monoclonal anti-HIF- $1 \alpha$ antibody (BD Transduction Laboratories), anti-mouse peroxidase-conjugated antibody (Sigma-Aldrich, St. Louis, MO, USA) and anti-goat peroxidase-conjugated antibody (Dako, Carpinteria, CA, USA).

CA9-specific shRNA oligonucleotides were first cloned into the pSUPER plasmid, which was then digested with ClaI and EcoRI. The resulting 300-bp fragment was excised, gelpurified and ligated into the EcoRI/ClaI-digested pLVTHM lentiviral vector to replace the pLVTHM H1 promoter with the H1 promoter and shRNA from pSUPER-shCA9. Recombinant pLVTHM-shCA9 was digested with FspI and $M s c \mathrm{I}$, and the 2.5-kb fragment was excised, purified and ligated into the inducible pLVET-tTR-KRAB lentiviral vector digested with $F s p \mathrm{I}$ and $M s c \mathrm{I}$. Plasmids pSPAX2 and pMD2G encoded packaging and envelope genes, respectively. A control plasmid bearing nonsense scramble shRNA ( $\mathrm{Scr}$ ) was constructed using the same method. All plasmids were obtained from the plasmid repository Addgene.com.

Production and titration of CA9-specific lentiviral particles. Lentiviral particles were produced in HEK 293T cells by co-transfection of the packaging plasmid $(2.9 \mu \mathrm{g})$, the virus envelope encoding plasmid $(1.2 \mu \mathrm{g})$, and the vector carrying both $C A 9$-shRNA (pLVET-shCA9, $4 \mu \mathrm{g}$ ) and the GFP transduction marker, using the GenePorterII transfection kit (Genlantis). Supernatant from the transfected cells was collected, filtered through a $0.22-\mu \mathrm{m}$ filter and either used directly for transduction or stored in aliquots at $-80^{\circ} \mathrm{C}$.

The virus was titrated by transducing HeLa cells with aliquots of viral stock in cultivation medium mixed with Polybrene $(5 \mu \mathrm{g} / \mathrm{ml})$. The transduced cells were incubated for 3 days in the presence of doxycycline and analyzed on the EasyCyte 6HT flow cytometer (Millipore) for expression of the GFP transduction marker. The titer was calculated from the number of plated cells multiplied by the proportion of transduced cells and divided by the volume of added viral stock.

Transduction of HT-1080 cells. The HT-1080 cells were mixed with the lentivirus particles at MOI of 10 and plated into 96-well plates. On the next day, the medium with doxycycline was replaced. Two days later, the cells were examined for
GFP expression and then seeded at a density of 500 cells per 100 -mm dish to form colonies in the presence of doxycycline. The colonies expressing GFP were picked up, expanded and used for further study.

Reverse transcription PCR. Total RNA was extracted using a GeneJET RNA Purification kit (Fermentas) and transcribed to cDNA using a High Capacity cDNA Reverse Transcription kit (Applied Biosystems). Quantitative PCR was performed with Power SYBR ${ }^{\circledR}$ Green PCR Master Mix on a StepOne RealTime PCR system (Applied Biosystems). Real-time PCR was performed using the following primers: MMP9 S, atcttccaa ggccaatcctact and A, ctatccagctcaccggtctc; MMP3 S, gttgttaggagaaaggacagtgg and A, tatgagcagcaacgagaaataa; Col4A1 $\mathrm{S}$, ggacaaarggtgatactgga and A, gccattgcatcctggaatac; Col4A2 S, agggtgaaaagggtgacgta and A, tgcctcttattcctggttcc; Itg $\beta 3 \mathrm{~S}$, acagtctgtgatgaaaagattgg and $\mathrm{A}$, cagcccaaagagggataat $\beta$-actin $\mathrm{S}$, ccaaccgcgagaagatgacc and $\mathrm{A}$, gatcttcatgaggtagtcagt.

Immunoblotting. The cells were grown for 3 days in medium containing doxycycline to induce $C A 9$ silencing. On the fourth day, the cells were moved into hypoxia for $24 / 48 \mathrm{~h}$ to induce expression of the CA IX protein. After incubation in hypoxia samples were lysed. Samples consisting of $100 \mu \mathrm{g}$ total proteins were separated in $10 \%$ SDS-PAGE and immunoblotting was performed as described elsewhere (11).

Immunofluorescence. Cells grown on glass coverslips with or without collagen coating were fixed in $4 \%$ paraformaldehyde and permeabilized with $0.1 \%$ Triton X-100. Immunofluorescence staining and confocal microscopy were carried out as previously described (10).

Microarray analysis. Affymetrix GeneChip Human Gene 1.0 ST (Affymetrix) was used for the microarray analysis following the standard protocol (starting with $100 \mathrm{ng}$ of total RNA). The analysis was performed in three replicates. The raw data were analyzed with Partek Genomics Suite (Partek Inc.). All hybridizations passed quality control. The data were background-corrected by the GC Robust Multi-Array Average (GC-RMA) algorithm, variance-stabilized by logarithmic transformation and normalized with the probeset level quantile method. One-way analysis of variance (ANOVA) was performed to identify genes varying significantly across two compared groups. Signaling Pathway Impact Analysis (SPIA) was performed using SPIA package of the Bioconductor within the R environment (http://www.r-project.org).

Spreading assay, wound healing assay and Matrigel invasion. Following treatment with doxycycline and exposure to hypoxia, HT-1080 cells were seeded on collagen-coated coverslips and left to attach for 15 and $30 \mathrm{~min}$, respectively. The cells were then fixed with 2\% paraformaldehyde in PBS for $10 \mathrm{~min}$ at room temperature (RT), washed with PBS, stained with $0.5 \%$ Coomassie blue for $5 \mathrm{~min}$ at RT and washed 3 times with PBS. Images of the fixed cells were analyzed using ImageJ software (http://rsb.info.nih.gov/ij/). At least 500 cells were analyzed for each sample, and cell-spreading areas of $C A 9$-silenced HT-1080 cells and relevant control samples were calculated and compared using the t-test. 
For the wound healing assay, HT-1080 cells were precultivated in hypoxia for $24 \mathrm{~h}$ with or without doxycycline. On the following day, the confluent monolayer was wounded with a sterile micropipette tip. The wound healing assay was performed in DMEM with $1 \%$ FCS containing $20 \mathrm{ng} / \mathrm{ml} \mathrm{HGF}$ in normoxic conditions, without the presence of doxycycline. The cells were photographed at 20 positions for each sample immediately after wound initiation and after $8 \mathrm{~h}$ using an inverted Zeiss microscope (Axiovert $40 \mathrm{CFL}$ ), 10x objective. Wound healing was quantified using ImageJ software (mean \pm standard deviation) and results were compared by the t-test $\left.{ }^{* *} \mathrm{P}<0.01\right)$.

Matrigel invasion was performed using BD Falcon HTS FluoroBlok $8.0-\mu \mathrm{m}$ colored PET membrane inserts for 24-well plates (BD Biosciences) using HT-1080 cells labeled with a lipophilic fluorescent dye DiO (Invitrogen) as previously described (10).

\section{Results}

Silencing of CA9. The CA IX protein plays an important role in hypoxic tumor cells as it helps them survive hostile conditions through efficient buffering of intracellular $\mathrm{pH}$ coupled with excretion of protons to the extracellular space. To elucidate CA IX-regulated molecular pathways, we investigated the effects of CA IX deficiency in tumor cells using an HT-1080 human fibrosarcoma model. These cells display hypoxia-inducible and density-dependent expression of CA IX (Fig. 1A), similarly as it was shown for many other tumor cell lines $(12,13)$. Conditional shRNA-mediated silencing of $C A 9$ was achieved using a tightly regulated pLVET-tTR-KRAB lentiviral vector, in which shRNA expression from the wildtype $\mathrm{H} 1$ promoter is governed by TTR-KRAB protein that epigenetically silences the integrated lentivirus in the absence of doxycycline (14). Several clonal cell lines targeting CA9 were selected according to immunoblotting analysis and were further studied in comparison with the scrambled (Scr) shRNA clones (Fig. 1C). Compared to the control Scr cells, the $C A 9$-silenced cells exposed to hypoxia showed a decreased extracellular acidification capacity (Fig. 1B), suggesting that they lost the pH-regulating function of CA IX and that our shRNA system was functional.

Gene expression profiling of the control vs. the CA9-silenced cells. To search for molecular signatures and pathways affected by CA IX protein deficiency, we performed pairwise gene expression profiling of the hypoxic cells bearing nonsense Scr shRNA vs. CA9 shRNA on the HT-1080 cell line background. Microarray experiments were carried out using three independent clones established from each of the CA9 shRNA and Scr shRNA cells. Differential gene expression analysis was performed by filtering the microarray data for genes that showed at least a 2 -fold change in gene expression levels in silenced vs. control cells $(\mathrm{P}<0.05)$.

By applying such selection criteria, the expression levels of 109 genes were found to be significantly altered. Among them, 62 genes were downregulated and 47 genes were upregulated in the $C A 9$-silenced cells compared to the Scr controls. The list of the 22 most highly differentially expressed genes is shown in Fig. 2A. Presence of $C A 9$ among them confirms efficiency
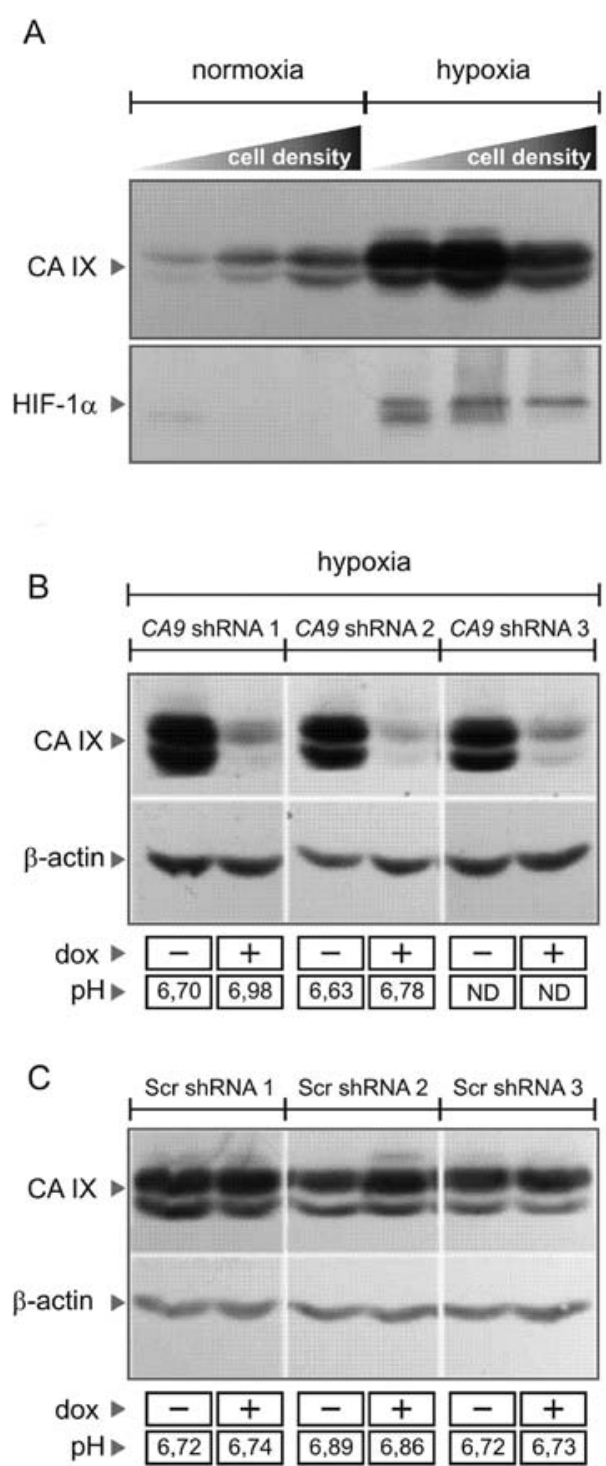

Figure 1. (A) Expression of endogenous CA IX and HIF-1 $\alpha$ proteins in HT-1080 cells grown at high and low density and under normoxic $\left(20 \% \mathrm{O}_{2}\right)$ and hypoxic conditions $\left(2 \% \mathrm{O}_{2}, 24 \mathrm{~h}\right)$. Increasing cell density induced CA IX expression while the hypoxic induction was associated with HIF-1 $\alpha$ protein accumulation. (B) Suppression of CA IX protein in HT-1080 CA9 shRNA 1, CA9 shRNA 2 and $C A 9$ shRNA 3 clonal cell lines was activated in the presence of doxycycline (dox, $0.5 \mu \mathrm{g} / \mathrm{ml}$ ). Extracellular $\mathrm{pH}$ measured in two parallel dishes of each CA9 shRNA clone was increased in the CA9-silenced cells. (C) Control clones of HT-1080 Scr shRNA 1, Scr shRNA 2 and Scr shRNA 3 bearing nonsense shRNA incubated in hypoxia with doxycycline (dox, $0.5 \mu \mathrm{g} / \mathrm{ml}$ ) or without displayed no change in CA IX protein level nor a change in extracellular $\mathrm{pH}$.

of silencing. Quantitative RT-PCR performed on two members of the matrix metalloproteinase family (MMP3 and MMP9) and collagen type IV $\alpha 2$, which are frequently associated with tumor phenotype, confirmed the microarray results (Fig. 2B). To identify molecular pathways deregulated upon $C A 9$ knockdown, we analyzed the microarray data using the Signaling Pathway Impact Analysis (SPIA) linked to KEGG (15). The analysis revealed inhibition of the pathways designated as small-cell lung cancer, focal adhesion and regulation of actin cytoskeleton in the $C A 9$-silenced cells. In contrast, pathways involved in cell cycle, RNA transport and protein processing in endoplasmic reticulum were found to be activated. SPIA 
A

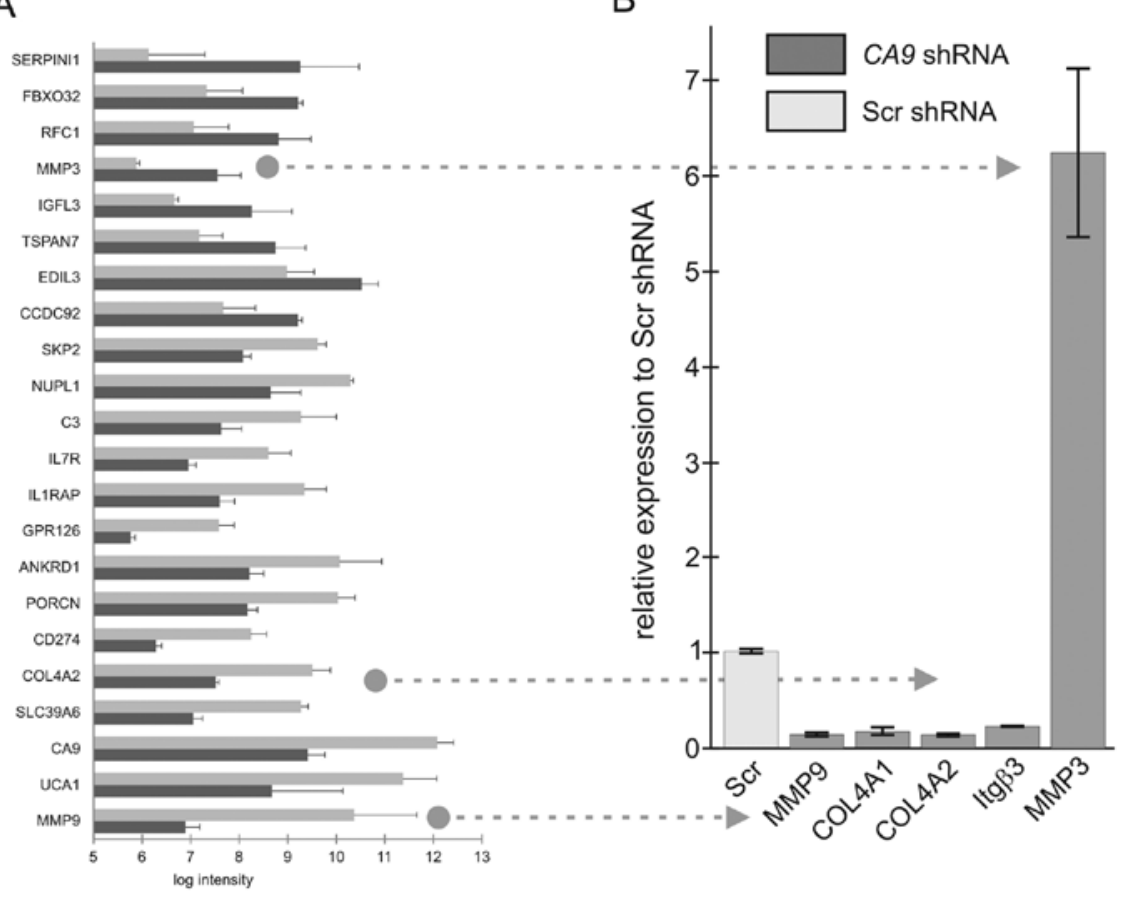

C SPIA two-way evidence plot

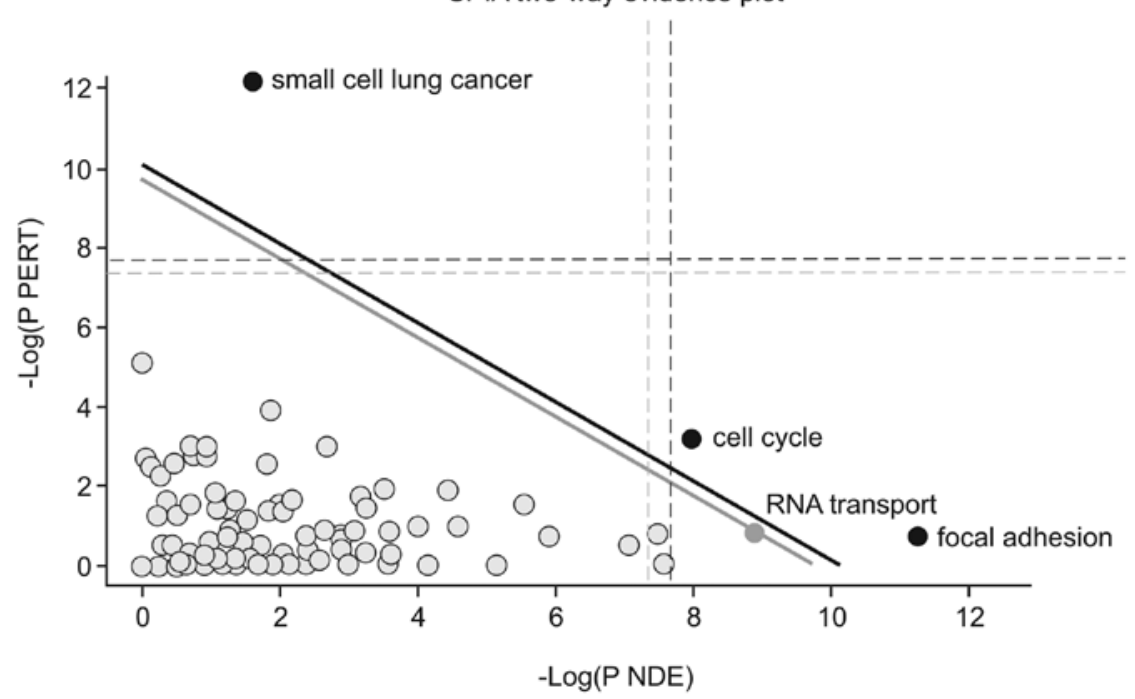

Figure 2. (A) Differential transcription of selected genes in Scr shRNA cells (light gray) and CA9 shRNA cells (dark gray). (B) Microarray results were confirmed by real-time PCR of the selected genes. Relative expression of genes related to the focal adhesion pathway in CA9-silenced compared to Scr cells was verified by real-time PCR. $\beta$-actin was used as an internal reference control. (C) SPIA analysis of differentially expressed genes. Analysis was performed on a set of differentially expressed genes with $\mathrm{P}<0.05$, $\mathrm{x}$-axis indicates enrichment of genes and $\mathrm{y}$-axis represents perturbation of genes within the pathway. Pathways above the gray line are significant at 0.05 after FDR correction and those above the black line at 0.05 after Bonferroni correction.

two-way evidence plot based on combining pPERT and pNDE indicated that the small-cell lung cancer, cell cycle and focal adhesion pathways were the most significantly affected (Fig. 2C).

Focal adhesion pathway is inhibited in the CA9-depleted cells. The ability of cells to form focal adhesions (FAs) as components of cell motility and migration represents an important early event in the development of metastasis. CA IX was previously shown to facilitate cell migration through its participation in the pH-regulating machinery driving lamellipodial extensions of moving cells (10). HT-1080 fibrosarcoma cells exhibited similar distribution of CA IX protein as in epithelial/carcinoma cells indicating that it might play an analogous role in the migration of tumor cells derived from fibrosarcoma (Fig. 3A). Importantly, microarray analysis revealed that the loss of CA IX resulted in downregulation of several molecules implicated in the interaction of cells with the extracellular matrix, such as integrin $\beta 3$, collagen type IV $\alpha 2$ and $\alpha 1$, dedicator of cytokinesis 9 (DOCK9), fermitin family member 2 (FERMT2), Rho-associated, and coiled-coil containing protein kinase 1 (ROCK1) (Fig. 3B). This finding 


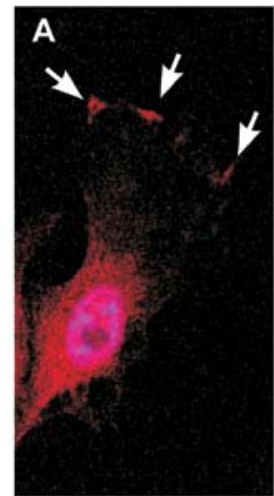

C

dox-
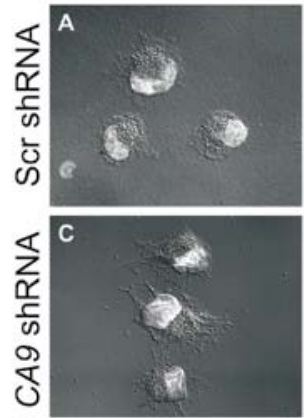

B $127 \square$ CA9 ShRNA

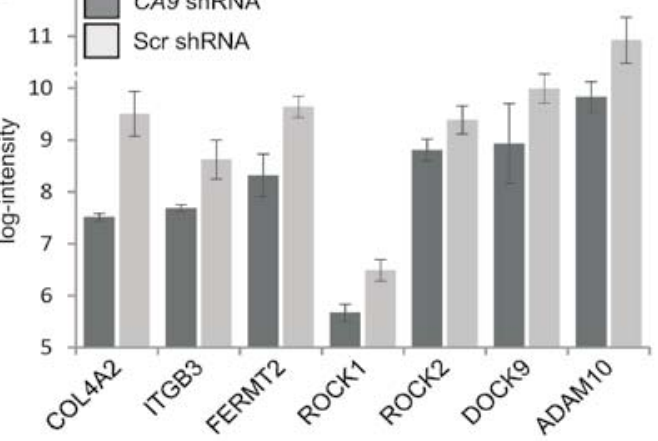

D

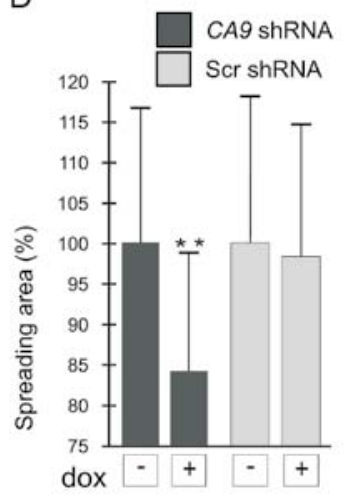

Figure 3. Loss of CA IX affects the focal adhesion pathway and reduces the spreading of HT-1080 cells. (A) Immunofluorescence analysis of wild-type HT-1080 fibrosarcoma cells exposed to hypoxia shows that CA IX is localized in lamellipodia. (B) Selection of cell-matrix interaction-associated genes deregulated by $C A 9$ silencing. (C) Spreading of scrambled and CA9 shRNA-transfected cells pre-incubated under hypoxia without (-dox) or with (+dox) doxycyclin and seeded in $0.5 \%$ FCS medium on a 12-well plate for $1 \mathrm{~h}$. Majority of CA IX-positive cells exhibited spreading while CA IX-deficient cells remained rounded. Nuclei of the cells were stained with DAPI. (D) Identically pre-incubated cells were plated on a collagen-coated surface and spreading was determined by measuring the increase in the cell area. Graph clearly shows the diminished spreading capacity of $C A 9$-silenced cells.

A

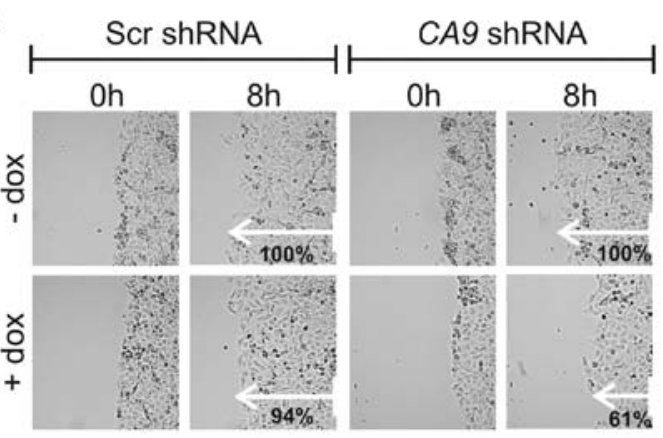

B

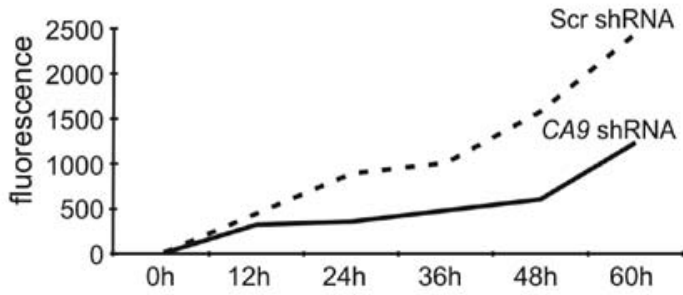

Figure 4. (A) Representative images of the wound healing assay captured immediately after scratching and after $8 \mathrm{~h}$. The cells were pre-incubated in hypoxia for $24 \mathrm{~h}$ with (+dox ) or without dox (-dox) as indicated. After scratching and HGF addition, the experiment was run in normoxia. A significant reduction in the migration rate (indicated by arrows) to $61 \%$ was noted in CA9 shRNA cells and no significant decrease was evident in the scrambled cells (as evaluated by $\mathrm{t}$-test, $\mathrm{n}=20, \mathrm{P}<0.01)$. The effect of CA IX on the migration rate was further demonstrated; no visible difference was noted among the CA9 shRNA cells without dox and the Scr shRNA cells with or without dox, all expressing a similar level of CA IX. (B) Effect of CA9 silencing on in vitro invasion through Matrigel. HT-1080 cells pre-incubated as described in Fig. 3C and D were plated on Matrigel-coated filters and allowed to invade in hypoxia for $72 \mathrm{~h}$. Knockdown of CA IX led to decreased invasive ability of HT-1080 cells.

suggests that CA IX affects additional aspects of cell migration, including FA assembly.

Therefore, we decided to further explore this assumption. We showed that CA IX-deficient cells were less capable of spreading on the support independently of collagen coating compared to control cells (Fig. 3C and D). In the wound healing assay, decreased expression of CA IX led to a significant reduction $(\mathrm{P}<0.01)$ in the migration rate to $61 \%$ in comparison to control cells (Fig. 4A). There was no significant difference in migration of scrambled cells with or without doxycycline, and between Scr and CA9 shRNA cells incubated without doxycycline. To assess the migratory and invasive abilities of the HT-1080 cells upon knockdown of $C A 9$, we performed a Matrigel invasion assay that mimics invasion of cells through a 
basement membrane. We observed reduced invasion/migration of CA IX-depleted cells (Fig. 4B). This could be related to the diminished level of MMP9, which is essential for invadopodia formation and cleavage of the basement membrane.

Altogether, these experiments confirmed the microarray predictions and further underlined the importance of $\mathrm{CA}$ IX in adhesion, migration and invasion of tumor cells under conditions of limited oxygen tension typical of the tumor microenvironment.

\section{Discussion}

Due to its tight transcriptional control by HIF-1 $\alpha$, CA IX is frequently used as a favored hypoxia marker in many types of tumors, where it can serve as a prognostic indicator and predictive factor $(5,16)$. Moreover, accumulating evidence suggests that CA IX plays an active role in tumor physiology principally through its catalytic activity-mediated control of $\mathrm{pH}$ and cell adhesion/migration (6,7). This evidence is mostly based on functional studies of various cellular models with CA IX overexpression, mutagenesis or suppression $(6,9,10,17-19)$. To date, only one study has reported the gene profiling of carcinoma cells with forced constitutive overexpression of CA IX. This study showed the involvement of CA IX in aberrant Rho-GTPase signal transduction leading to decreased cell adhesion and augmented cell motility (20).

In the present study, we employed a different approach, using inducible CA9 mRNA silencing in HT-1080 fibrosarcoma cells . Efficiency of silencing was demonstrated by the abrogation of the capacity of CA IX to acidify culture media, in line with the data obtained in a colon adenocarcinoma cell model (17).

Gene expression profiling of hypoxic HT-1080 cells revealed that 109 genes were significantly deregulated as a result of the loss of CA IX. These genes were members of several cancer-related pathways, with the prominent pathway designated as 'focal adhesion' that involves components of cell adhesion-migration-invasion machinery which was inhibited in CA IX-depleted cells.

Focal adhesion mediates cell-extracellular matrix interactions, which are essential for migration and invasion of tumor cells of both epithelial and fibroblast origin. It is a complex process that comprises more than 50 proteins from the inner and outer side of the cell membrane (21). Two members of the type IV collagen gene family namely COL4A1 and COL4A2 were among the most downregulated genes within the identified FA pathway. Both are structural constituents of the extracellular matrix (ECM) important for proper ECM assembly. Integrin $\beta 3$, a receptor for fibronectin, fibrinogen, plasminogen, prothrombin, thrombospondin and vitronectin, was also downregulated in the CA9-silenced HT-1080 cells. One of the FA family members downregulated in our study was FERMT2, which functions in cell shape modulation (22). Regulation of FA is also mediated by intracellular signaling from the Rho family of small GTPases to ROCK-I/ROCK-II kinases (23). Both molecules are responsible for actomyosin contractility, which is important for cellular movement. It is notable that both kinases were downregulated in the CA9-silenced cells. Another signaling molecule negatively affected by CA IX deficiency was DOCK9 also known as Zizimin1 (24). It is a member of the DOCK family of guanine nucleotide exchange factors (25). DOCK9 is an activator of $\mathrm{Cdc} 42$ which regulates the actin cytoskeleton, cell migration and several other cellular activities.

Downregulation of the above-mentioned genes was clearly mirrored by significantly reduced adhesion and spreading of CA IX-deficient HT-1080 cells. This resembles formation of aberrant FA in cells expressing the inactive NHE1 transporter (normally responsible for export of protons) as well as in cells lacking an MCT4 lactate transporter $(26,27)$. All of these three proteins are known to support cell migration by ensuring a proper $\mathrm{pH}$ gradient across the membranes of lamellipodia (28). It seems plausible that the $\mathrm{pH}$-regulating function is important also for assembly and/or disassembly of FAs, which is a ratelimiting process for efficient cell migration accompanying lamellipodial extension and retraction.

CA IX-deficient cells also exhibited reduced capacity for invasion and migration through Matrigel. This could be due to the fact that several FA-related genes, downregulated in the absence of CA IX, also participate in cell invasion, such as integrin subunits $\beta 3$ and $\alpha 6$ (29), and matrix metalloproteinase MMP9 (30). In contrast, MMP2 and MMP3 were upregulated in the $C A 9$-silenced cells. It is well known that protonation of MMP3 itself and protonation of fibrinogen as a substrate for MMP2 influence their activity and proteolysis (31-33). Since CA IX contributes to acidic pericellular $\mathrm{pH}$ by generation of extracellular protons, its loss may lead to reduced MMP2 and MMP3 activity despite their increased levels. Furthermore, MMP2-mediated collagenolysis depends on MMP2 interaction with $\beta 3$ integrin $(34,35)$, and thus lower $\beta 3$ integrin subunit expression can contribute to MMP2 inactivation and reduced invasion of CA IX-deficient cells. An additional molecule downregulated in the CA9-silenced HT-1080 cells potentially affecting their adhesion and invasion was ADAM10, which acts through shedding of many cell surface molecules including the key cell-cell adhesion molecule E-cadherin (36).

Altogether, our study provided the first molecular insight into downstream signal transduction pathways driven by CA IX in tumor cells using an inducible shRNA-based silencing approach. In accordance with independent functional studies, CA IX-emitted signaling was found to be important in several key aspects of cell adhesion, migration and invasion mediated by molecules that are central players in these phenomena. In addition to the known CA IX effects on destabilization of cell-cell contacts and stimulation of epithelial cell motility/migration, we demonstrated here for the first time its direct role in focal adhesion and invasion of tumor fibroblasts. This suggests that CA IX operates at different stages of the metastatic cascade and in various cell types. Notably, both direct and indirect evidence supports the view that CA IX acts at each stage via its catalytic activity-mediated $\mathrm{pH}$ regulatory capacity, suggesting that $\mathrm{pH}$ regulation is a critical facet of the cancer phenotype. Thus, our observation that CA IX has an impact on the pro-metastatic behavior of tumor cells supports recent efforts to explore its suppression or pharmacologic inhibition for anticancer therapy.

\section{Acknowledgements}

This study was supported by grants no. 2/0194/09 and 2/0130/11 from the Scientific Grant Agency of the Ministry 
of Education of the Slovak Republic and the Slovak Academy of Sciences, from the Research and Development Support Agency (contracts APVV-0108-10 and DO-7RP-0017-09) and from EU (7FP Collaborative project METOXIA) and from the Research and Development Operational Program funded by ERDF (project PV-INF-PAT, ITMS 26240220032).

\section{References}

1. Harris AL: Hypoxia - a key regulatory factor in tumour growth. Nat Rev Cancer 2: 38-47, 2002.

2. Pastorekova S, Parkkila S, Pastorek J and Supuran CT: Carbonic anhydrases: current state of the art, therapeutic applications and future prospects. J Enzyme Inhib Med Chem 19: 199-229, 2004.

3. Pastorek J, Pastorekova S, Callebaut I, et al: Cloning and characterization of $\mathrm{MN}$, a human tumor-associated protein with a domain homologous to carbonic anhydrase and a putative helixloop-helix DNA binding segment. Oncogene 9: 2877-2888, 1994.

4. Wykoff CC, Beasley NJ, Watson PH, et al: Hypoxia-inducible expression of tumor-associated carbonic anhydrases. Cancer Res 60: 7075-7083, 2000.

5. Potter $\mathrm{C}$ and Harris AL: Hypoxia inducible carbonic anhydrase IX, marker of tumour hypoxia, survival pathway and therapy target. Cell Cycle 3: 164-167, 2004.

6. Svastova E, Hulikova A, Rafajova M, et al: Hypoxia activates the capacity of tumor-associated carbonic anhydrase IX to acidify extracellular pH. FEBS Lett 577: 439-445, 2004

7. Swietach P, Hulikova A, Vaughan-Jones RD and Harris AL: New insights into the physiological role of carbonic anhydrase IX in tumour pH regulation. Oncogene 29: 6509-6521, 2010.

8. Raghunand N, Gatenby RA and Gillies RJ: Microenvironmental and cellular consequences of altered blood flow in tumours. Br J Radiol 76 Spec No 1: S11-S22, 2003.

9. Svastova E, Zilka N, Zat'ovicova M, et al: Carbonic anhydrase IX reduces E-cadherin-mediated adhesion of MDCK cells via interaction with beta-catenin. Exp Cell Res 290: 332-345, 2003.

10. Svastova E, Witarski W, Csaderova L, et al: Carbonic anhydrase IX interacts with bicarbonate transporters in lamellipodia and increases cell migration via its catalytic domain. J Biol Chem 287: 3392-3402, 2012.

11. Takacova M, Holotnakova T, Barathova M, Pastorekova S, Kopacek J and Pastorek J: Src induces expression of carbonic anhydrase IX via hypoxia-inducible factor 1 . Oncol Rep 23 869-874, 2010.

12. Ihnatko R, Kubes M, Takacova M, et al: Extracellular acidosis elevates carbonic anhydrase IX in human glioblastoma cells via transcriptional modulation that does not depend on hypoxia. Int J Oncol 29: 1025-1033, 2006.

13. Kaluz S, Kaluzova M and Stanbridge EJ: Expression of the hypoxia marker carbonic anhydrase IX is critically dependent on SP1 activity. Identification of a novel type of hypoxia-responsive enhancer. Cancer Res 63: 917-922, 2003.

14. Szulc J, Wiznerowicz M, Sauvain MO, Trono D and Aebischer P: A versatile tool for conditional gene expression and knockdown Nat Methods 3: 109-116, 2006.

15. Tarca AL, Draghici S, Khatri P, et al: A novel signaling pathway impact analysis. Bioinformatics 25: 75-82, 2009.

16. Pastorekova S, Ratcliffe PJ and Pastorek J: Molecular mechanisms of carbonic anhydrase IX-mediated $\mathrm{pH}$ regulation under hypoxia. BJU Int 101 (Suppl 4): 8-15, 2008
17. Chiche J, Ilc K, Laferriere J, et al: Hypoxia-inducible carbonic anhydrase IX and XII promote tumor cell growth by counteracting acidosis through the regulation of the intracellular $\mathrm{pH}$ Cancer Res 69: 358-368, 2009.

18. Hulikova A, Zatovicova M, Svastova E, et al: Intact intracellular tail is critical for proper functioning of the tumor-associated, hypoxia-regulated carbonic anhydrase IX. FEBS Lett 583: 3563-3568, 2009.

19. Ditte P, Dequiedt F, Svastova E, et al: Phosphorylation of carbonic anhydrase IX controls its ability to mediate extracellular acidification in hypoxic tumors. Cancer Res 71: 7558-7567, 2011.

20. Shin HJ, Rho SB, Jung DC, Han IO, Oh ES and Kim JY: Carbonic anhydrase IX (CA9) modulates tumor-associated cell migration and invasion. J Cell Sci 124: 1077-1087, 2011.

21. Zamir E and Geiger B: Molecular complexity and dynamics of cell-matrix adhesions. J Cell Sci 114: 3583-3590, 2001.

22. Tu Y, Wu S, Shi X, Chen K and Wu C: Migfilin and Mig-2 link focal adhesions to filamin and the actin cytoskeleton and function in cell shape modulation. Cell 113: 37-47, 2003.

23. Sanz-Moreno V, Gadea G, Ahn J, et al: Rac activation and inactivation control plasticity of tumor cell movement. Cell 135: 510-523, 2008

24. Meller N, Irani-Tehrani M, Kiosses WB, Del Pozo MA and Schwartz MA: Zizimin1, a novel Cdc42 activator, reveals a new GEF domain for Rho proteins. Nat Cell Biol 4: 639-647, 2002.

25. Cote JF and Vuori K: Identification of an evolutionarily conserved superfamily of DOCK180-related proteins with guanine nucleotide exchange activity. J Cell Sci 115: 4901-4913, 2002.

26. Gallagher SM, Castorino JJ and Philp NJ: Interaction of monocarboxylate transporter 4 with beta1-integrin and its role in cell migration. Am J Physiol Cell Physiol 296: C414-C421, 2009.

27. Denker SP and Barber DL: Cell migration requires both ion translocation and cytoskeletal anchoring by the $\mathrm{Na}-\mathrm{H}$ exchanger NHE1. J Cell Biol 159: 1087-1096, 2002.

28. Stock C and Schwab A: Protons make tumor cells move like clockwork. Pflugers Arch 458: 981-992, 2009.

29. Jin $\mathrm{H}$ and Varner J: Integrins: roles in cancer development and as treatment targets. Br J Cancer 90: 561-565, 2004.

30. Nagase H, Visse R and Murphy G: Structure and function of matrix metalloproteinases and TIMPs. Cardiovasc Res 69: $562-573,2006$

31. Rupp PA, Visconti RP, Czirok A, Cheresh DA and Little CD: Matrix metalloproteinase 2-integrin alpha(v)beta3 binding is required for mesenchymal cell invasive activity but not epithelial locomotion: a computational time-lapse study. Mol Biol Cell 19: 5529-5540, 2008.

32. Holman CM, Kan CC, Gehring MR and Van Wart HE: Role of His-224 in the anomalous $\mathrm{pH}$ dependence of human stromelysin-1. Biochemistry 38: 677-681, 1999.

33. Monaco S, Gioia M, Rodriguez J, et al: Modulation of the proteolytic activity of matrix metalloproteinase-2 (gelatinase A) on fibrinogen. Biochem J 402: 503-513, 2007.

34. Brooks PC, Stromblad S, Sanders LC, et al: Localization of matrix metalloproteinase MMP-2 to the surface of invasive cells by interaction with integrin alpha $\mathrm{v}$ beta 3 . Cell 85: 683-693, 1996.

35. Hornebeck W, Emonard H, Monboisse JC and Bellon G: Matrix-directed regulation of pericellular proteolysis and tumor progression. Semin Cancer Biol 12: 231-241, 2002.

36. Maretzky T, Reiss K, Ludwig A, et al: ADAM10 mediates E-cadherin shedding and regulates epithelial cell-cell adhesion, migration, and beta-catenin translocation. Proc Natl Acad Sci USA 102: 9182-9187, 2005. 